\title{
Aggregation over Metric Spaces: Proposing and Voting in Elections, Budgeting, and Legislation
}

\author{
Laurent Bulteau \\ LIGM, CNRS, Univ Gustave Eiffel \\ 5 Bd Descartes, 77454 Marne la Vallée, France \\ Gal Shahaf \\ Ehud Shapiro \\ Weizmann Institute of Science \\ Herzl St 234, Rehovot, Israel \\ Nimrod Talmon \\ Ben-Gurion University \\ Ben-Gurion Boulevard, Be'er Sheva, Israel
}

LAURENT.BULTEAU@U-PEM.FR

GAL.SHAHAF@WEIZMANN.AC.IL

TALMONN@BGU.AC.IL

\author{
EHUD.SHAPIRO@WEIZMANN.AC.IL
}

\begin{abstract}
We present a unifying framework encompassing a plethora of social choice settings. Viewing each social choice setting as voting in a suitable metric space, we offer a general model of social choice over metric spaces, in which - similarly to the spatial model of elections - each voter specifies an ideal element of the metric space. The ideal element acts as a vote, where each voter prefers elements that are closer to her ideal element. But it also acts as a proposal, thus making all participants equal not only as voters but also as proposers. We consider Condorcet aggregation and a continuum of solution concepts, ranging from minimizing the sum of distances to minimizing the maximum distance. We study applications of our abstract model to various social choice settings, including single-winner elections, committee elections, participatory budgeting, and participatory legislation. For each setting, we compare each solution concept to known voting rules and study various properties of the resulting voting rules. Our framework provides expressive aggregation for a broad range of social choice settings while remaining simple for voters; and may enable a unified and integrated implementation for all these settings, as well as unified extensions such as sybil-resiliency, proxy voting, and deliberative decision making.
\end{abstract}

\section{Introduction}

As social choice theory studies different settings of preference aggregation, correspondingly, there are different social choice models for these different settings in the literature on social choice. For example, the most basic social choice setting is single-winner elections - see, e.g., the books of Arrow (1951), Sen (1986), and Duncan (1958), in which voter preferences over a given set of alternatives are aggregated to decide upon a single winning alternative. More complex social choice settings include multiwinner elections (Faliszewski, Skowron, Slinko, \& Talmon, 2017b), in which voter preferences are aggregated to select a winning committee, and participatory budgeting (Goel, Krishnaswamy, Sakshuwong, \& Aitamurto, 2015; Aziz, Lee, \& Talmon, 2018b; Faliszewski \& Talmon, 2018; Aziz \& Lee, 2019), in which voter preferences are aggregated to select a bundle of items respecting a given budget 
limit. Further social choice settings include graph aggregation (Endriss \& Grandi, 2017) and aggregation of combinatorial domains (Chevaleyre, Endriss, Lang, \& Maudet, 2008).

Indeed, usually, mathematical foundations and theories in the social choice literature are tailored for a specific setting. Here, however, we study a general framework of social choice that can be applied to many settings at once. Why do we develop such a framework?

First, observing the plethora of social choice settings, one merit of our approach is that it incorporates many such settings uniformly; as such, it allows for an investigation of complex settings and for convenient comparisons between different social choice settings.

Second, such a broad and all-encompassing approach may enable a unified and integrated implementation to many settings at once. For example, if one is to develop a practical system allowing humans to act as voters, then one might use the same graphical user interface for many social choice settings at once. Furthermore, such a unified approach may allow for incorporating several orthogonal aspects of social choice that are relevant to different settings of social choice, at once; for example, one may develop Sybil-resilient aggregation methods that are applicable to all the social choice settings that can be incorporated in our unified model.

A third motivation for this work comes from Reality-Aware Social Choice (Shapiro \& Talmon, 2018), which requires the status quo (Reality) to always be present as a distinguished alternative when electing among several alternatives. According to Reality-Aware Social Choice, if the majority perceives the status quo as negative and prefers a particular alternative to the status quo, then that alternative will be chosen. If the majority prefers the status quo over every proposed alternative, then it will be chosen. But if the majority prefers other alternatives to the status quo, but no specific alternative among those proposed has majority support, then the status quo reigns. This situation is clearly distressing, as the will of the people to change the status quo cannot be accommodated, perhaps due to the incompetence or interests of those who select the alternatives to vote upon, or the lack of an adequate mechanism to identify alternatives that may have majority support over the status quo. The framework we develop in this paper proposes a way to identify such alternatives; this is so as, in essence, the set of alternatives to vote on does not have to be a-priori set but the designer of the election, but voters can propose alternatives, even when the space of possible alternatives is infinite. Put differently, a key merit of our approach is in it being egalitarian, in the sense that participants are not just equal as voters on proposals predetermined by others, but are also equal as proposers. This is manifest especially in our approach to budgeting and legislation, where the domains of budgets and laws are open-ended and potentially infinite. This is in contrast to the classical social choice setting, in which an external authority is assumed to fix a finite number of alternatives prior to the vote. Thus, our model may enable a deliberative process in which participants transition between making proposals, negotiating changes to competing proposals to achieve common ground, building coalitions in support of a particular proposal, and supporting proposals different from their original ones as a result of negotiation, deliberation, and coalition building. A first step in this direction was taken recently (Elkind, Grossi, Shapiro, \& Talmon, 2020), where a deliberative framework in which participants are equal as proposers, deliberators, coalition builders, and voters is studied. A key step in this deliberative framework is for two coalitions to find a workable compromise proposal; the aggregation methods developed herein could be used to help identify such compromises. 
A fourth motivation for our work is to be able to incorporate very complex aggregation scenarios - such as participatory legislation - while remaining relatively simple for the voters. Indeed, this is done by letting voters only specify their ideal elements, and relying on a metric space to infer more complex preferences. On the other hand, we indeed acknowledge that this simplicity means that certain voter preferences cannot be expressed, such as a dependency among budget items or the inconsistency of an intermediate element between two legislative texts; we elaborate on this point in Section 10 and discuss possible remedies. For now, we stress that a key restriction of our model is that voters specify ideal points from some space and the resulting aggregated solution is also an element of the space. We believe that, even though this is indeed a restriction, the model still captures many real-world settings in a convenient way.

A specific motivating scenarios is that of digital democracy (Shapiro, 2018). In particular, in a digital democracy, the community has to address many types of decisions; e.g., the choice of officers, committees, parameters (e.g., interest rate), budgets, and legislation. To be able to address all these issues - that are, essentially, different social choice settings - in a unified and convenient way, it is useful to have a single, broad model that can encompass all of these settings at once.

Finally, from a more technical point of view, our study sheds light on issues that relate to the possibility of finding efficient aggregation algorithms to many of the scenarios we consider. While here we concentrate on the theoretical foundations of our framework, coping with the NP-hardness of the remaining scenarios is left for future study.

Our model, described in Section 3, consists of elements connected via a metric. The elements of the metric space may serve in three roles: proposals, votes, and outcomes of the vote aggregation process. We associate each voter with a single element of the space, referred to as the voter's ideal element, understood as her most-preferred element of the space. Then, we assume that each voter prefers closer elements to elements which are farther away from her ideal element. Given a metric space, we are interested in aggregating several voter ideal elements in it into an aggregated element, which is the result of the election (we discuss other options in Section 10.4). We study several solution concepts: Condorcet aggregation, in which a Condorcet-winning aggregated element is selected (if it exists); and a continuum of solution concepts, containing on one end minimizing the sum of distances to the ideal elements and, at the other end, minimizing the maximum distance to the ideal elements.

We study applications of this model to various settings of social choice. For each setting we identify a metric space suitable for the application, and investigate each solution concept by comparing it to known aggregation methods and studying its axiomatic properties. While our model can be applied to many social choice settings, here we concentrate on the following, prominent ones:

1. Plurality elections (Section 4);

2. Single-winner elections over a 1-dimensional Euclidean issue space (Section 5);

3. Continuous participatory budgeting (Section 6);

4. Social welfare functions (Section 7); 
5. Committee elections (Section 8); and

6. Participatory legislation (Section 9), in which the task is to aggregate text drafts.

The study of further settings is left to future work. A key initial motivation for our work was participatory legislation, which is perhaps the most complex setting we study here. To the best of our knowledge, here we propose the first aggregation method for this important social choice setting.

\section{Related Work}

The spatial model of elections (Enelow \& Hinich, 1984) considers voter ideal elements and infers voter preferences using an underlying Euclidean metric. This model was later extended to Hilbert spaces (Gershkov, Moldovanu, \& Shi, 2019), normed spaces (Peters, van der Stel, \& Storcken, 1993), and semi-inner product spaces (Gershkov, Moldovanu, \& Shi, 2020). Here, we consider general metric spaces, including discrete and not limited to complete and well-structured ones. This approach allows us to apply this model to a broad range of social choice settings, including elections, budgeting, committee elections and legislation.

Metric preferences are quite prominent in the social choice literature. Much of this research - including that regarding spatial voting games (Filtser \& Filtser, 2020) - is similar to ours in considering ideal elements of voters and alternatives. Contrary to our model, however, extant literature is usually concerned with aggregating derived rankings rather than with directly aggregating the ideal elements themselves. Specifically, the notion of distortion (Procaccia \& Rosenschein, 2006) is prominent (Goel, Krishnaswamy, \& Munagala, 2017; Skowron \& Elkind, 2017; Anshelevich, Bhardwaj, Elkind, Postl, \& Skowron, 2018). Here, in contrast, we aim to aggregate the ideal elements and argue this to be a productive approach. Indeed, the idea of using distances in social choice is prominent; e.g., distance-based completion principles used in combinatorial voting (Lang \& Xia, 2016) which relate to set extensions (Barbera, Bossert, \& Pattanaik, 2004) and are used in committee elections. We do not aim to reinvent the wheel in the applications we consider, but rather to propose a unified model and incorporate many social choice settings at once; thus, we discuss such relations in the respective sections.

We do mention the general concept of distance rationalizability (DR) (Elkind, Faliszewski, \& Slinko, 2015), in which voting rules are defined via consensus classes and distances. Here, we do not consider consensus classes per se, but aggregation methods of ideal elements. Specifically, DR is concerned with summing voter distances to reach consensus in the ordinal model of single-winner elections. This relates to our social choice maximization - specifically, the unanimous consensus (Elkind et al., 2015). But, notions such as Condorcet aggregation do not easily fit to DR and, furthermore, our focus is on a general metric-based model as it applies to a variety of social choice settings.

We also mention the work of Feldman et al. (2016); specifically, their location model is very similar to our metric-based model, but their study is focused on other aspects, such as on randomized mechanisms. We also mention the work of Fain et al. (2017) and Garg et al. (2017), which also consider aggregation in continuous metric spaces. Additional works that tackle facility location mostly focus on strategic voting over simple metric spaces as the 
line or the circle (Procaccia \& Tennenholtz, 2009; Meir, Procaccia, \& Rosenschein, 2012; Anshelevich \& Postl, 2017).

Finally, we view our model as egalitarian wrt. proposals as each agent can propose their own proposal; in this context we refer also to the work of Elkind et al. (2020) that describes a similar egalitarian process. We mention work on citizen-candidates in this context (Osborne \& Slivinski, 1996) as that line of work can be viewed as egalitarian in the sense that each citizen can be a candidate.

\section{Preliminaries}

Here we first describe the general model, then describe several aggregation methods and axiomatic properties. We end this section with an overview of our results.

\subsection{Formal Model}

We describe the formal ingredients of our model.

Metric Spaces Our model consists of a metric space $(X, d)$, where $X$ is a set of elements and $d: X \times X \rightarrow \mathbb{R}$ is a metric function. Namely (1) $d$ is symmetric, with $d(x, y)=d(y, x)$ for every pair $x, y \in X,(2) d$ is non-negative, with $d(x, y) \geq 0$ and $d(x, y)=0$ if and only if $x=y$, and (3) $d$ satisfies the triangle inequality, i.e., $d(x, z) \leq d(x, y)+d(y, z)$ holds for all $x, y, z \in X$.

Voters, Ideal Elements, and Inferred Weak Orders We assume $n$ voters, where voter $i$ corresponds to an element $v_{i} \in X$. We refer to $v_{i}$ as the ideal element of voter $i$, interpreted as her most-preferred element of the metric space. Based on the ideal element $v_{i}$, and the metric itself, we infer a ranking over the whole metric space, where, for each two elements $x, y \in X$, voter $i$ prefers $x$ to $y$ (i.e., ranks $x$ higher than $y$ ) if and only if $d\left(v_{i}, x\right)<d\left(v_{i}, y\right)$; we denote it by $x \succ_{i} y$. A voter is indifferent to elements $x$ and $y$ for which $d\left(v_{i}, x\right)=d\left(v_{i}, y\right)$. Slightly overloading the notation, we use $v_{i}$ to denote both the ideal element and this inferred weak ranking of voter $i$ (the two cases will always be clear from the context).

\subsection{Aggregation Methods}

With respect to some metric space $(X, d)$, an aggregation method is a function $\mathcal{R}: X^{n} \rightarrow$ $P(X)$ that takes as input $n$ ideal elements $V=\left\{v_{1}, \ldots, v_{n}\right\}$ of the voters and returns a non-empty set of aggregated elements. We refer to $\mathcal{R}(V)$ as the co-winners according to $\mathcal{R}$ of the election corresponding to $V$ over the metric space $(X, d)$.

Condorcet Aggregation We adapt the Condorcet principle to our model.

Definition 1 (Condorcet winner). Let $(X, d)$ be a metric space and consider $n$ voters with their ideal elements and corresponding weak rankings over $X$, denoted by $V=\left\{v_{1}, \ldots, v_{n}\right\}$. An element $x \in X$ is a Condorcet winner wrt. $V$ if for any other element $y \in X \backslash\{x\}$, it holds that $\left|\left\{v_{i} \in V: x \succ_{i} y\right\}\right|>\left|\left\{v_{i} \in V: y \succ_{i} x\right\}\right|$.

That is, a Condorcet-winning element is such that, if chosen, then there is no voter majority for changing it. For simplicity, we shall use the notation $V(x \succ y):=\mid\left\{v_{i} \in\right.$ 
$\left.V: x \succ_{i} y\right\} \mid$, under which $x \in X$ is a Condorcet winner if $V(x \succ y)>V(y \succ x)$ for every $y \in X \backslash\{x\}$. An aggregation method $\mathcal{R}$ is Condorcet-consistent if $\mathcal{R}(V)$ is the set of Condorcet winners for every set of votes $V .^{1}$ Throughout, we refer to Condorcet aggregation as an aggregation method outputting the Condorcet winner, if it exists, or outputting nil otherwise.

$\boldsymbol{L}_{\boldsymbol{p}}$ Aggregation Besides Condorcet aggregation, we consider $L_{p}$ aggregation, a continuum of solution concepts, parameterized by a parameter $1 \leq p \leq \infty$. Specifically, for a given $p$, we look for an element which minimizes the sum of $p$-factored distances to the voter ideal elements.

Definition 2. Let $(X, d)$ be a metric space and consider $n$ voters with their ideal elements, denoted by $V=\left\{v_{1}, \ldots, v_{n}\right\}$. For $p \neq \infty$, the $L_{p}$ estimator of $V$ is defined by ${ }^{2}$

$$
L_{p}(V):=\underset{x \in X}{\arg \min } \sum_{i \in[n]} d\left(x, v_{i}\right)^{p}
$$

For $p=\infty$, we define

$$
L_{\infty}(V):=\underset{x \in X}{\arg \min } \max _{i \in[n]}\left\{d\left(x, v_{i}\right)\right\} .
$$

The $L_{p}$ aggregation method returns these elements $x$ as the co-winners.

Slightly abusing terminology, we sometimes speak of algorithms that "minimize $L_{p}$ " to mean that they select an element that has the minimum $p$-factored distances to the voter ideal points.

Note that $L_{p}(V)$ is not necessarily unique, thus we treat this term as a subset of $X$. (Furthermore, $L_{p}(V)$ might be empty.) To tackle the issue of uniqueness, we sometimes consider reduced $L_{p}$ aggregation:

$$
\widetilde{L_{p}}(V):=\lim _{\substack{p \neq q \\ q \rightarrow p}} L_{q}(V)
$$

that satisfies $\widetilde{L_{p}}(V) \subseteq L_{p}(V)$ due to the continuity of $\sum_{i \in[n]} d\left(v_{i}, x\right)^{p}{ }^{3}$ These notions would mainly be applied to resolve uniqueness issues for $p \in\{1, \infty\}$.

$L_{p}$ norm estimators have been widely studied in the statistical literature (Sposito, 1990), and are usually applied when estimating the parameters in linear regression models. The choice of the parameter $p$ in this context critically depends on the underlying distribution, and reflects a tradeoff between the number of samples needed (the efficiency of the estimator) and its sensitivity to departures from normality in the residual distribution (the estimator's robustness) (Pennecchi \& Callegaro, 2006).

To better understand the notion of $L_{p}$ estimation, observe that $L_{1}(V)$ minimizes the sum of absolute errors, $L_{2}(V)$ minimizes the mean squared error, and $L_{\infty}(V)$ minimizes

1. Weak Condorcet winners are defined similarly, by replacing $>$ with $\geq$ in the definition above. As the distinction between Strong/Weak Condorcet winners is in essence an issue of tie breaking, we will not focus on it.

2. For some $n \in \mathbb{N},[n]=\{1, \ldots, n\}$.

3. Note that the limit in the definition of $\widetilde{L_{p}}(V)$ is taken over sets, i.e., $x \in \widetilde{L_{p}}(V)$ if there is a sequence $x_{n} \rightarrow x$ with $x_{n} \in L_{q_{n}}(V)$ and $q_{n} \rightarrow p$. 
the maximal distance from all the ideal elements. ${ }^{4} L_{p}$ norms have been also studied within the social choice literature (Faliszewski, Skowron, Slinko, \& Talmon, 2017a). Intuitively, in the context of vote aggregation, the choice of the parameter $p$ reflects the relative influence of an individual in a joint decision making. For example, applying $L_{\infty}$ as a voting rule, intuitively, protects minority rights, in the sense that it does not disregard outliers (in this context we mention the work of Aziz et al. (2018a) who use the maximum operator to achieve the same protection for minorities).

\subsection{Axiomatic Properties}

In subsequent sections we consider applications of our general model. To better understand how different solution concepts behave in each application, we consider several axiomatic properties.

Definition 3. An aggregation method $\mathcal{R}$ is majoritarian if, whenever a strong voter majority (i.e., strictly more than half of the voters) selects the same ideal element $w$, then $w$ is selected by the aggregation method; formally, $\mathcal{R}$ is majoritarian if $\left|\left\{v_{i} \in V: v_{i}=w\right\}\right|>|V| / 2$ implies that $w \in \mathcal{R}(V)$.

Definition 4. An aggregation method $\mathcal{R}$ is monotone if, for each set $V=\left\{v_{1}, \ldots, v_{n-1}, v\right\}$ of votes and for each co-winner $w \in \mathcal{R}(V)$ it holds that $w \in \mathcal{R}\left(V^{\prime}\right)$, where $V^{\prime}=\left\{v_{1}, \ldots, v_{n-1}, v^{\prime}\right\}$ and $v^{\prime}=w$.

That is, majoritarity holds if the aggregated element equals the majority ideal element, while monotonicity holds if moving an ideal element to the aggregated element does not change the aggregated element.

\subsection{Overview of our Results}

Throughout, we study properties of Condorcet aggregation and $L_{p}$ aggregation for certain applications of our general model. We note that distance metrics may not be sufficiently expressive in general, as they cannot express dependencies (among candidates or budget items) or semantic relations (among sentences in a legislation). Related, in our model we implicitly assume that voters with the same ideal point have exactly the same preferences wrt. all elements of the metric space. Overcoming these limitations is the topic of future research. ${ }^{5}$ Table 1 lists our main results.

4. In the statistical context there is an underlying assumption of some unknown distribution; this corresponds to objective social choice, in which the goal of voting rules is to recover some assumed ground truth (Brandt, Conitzer, Endriss, Procaccia, \& Lang, 2016). Here we are interested in subjective social choice, in which the goal of voting rules is to aggregate individual opinions of agents (Caragiannis, Nath, Procaccia, \& Shah, 2017); thus, we do not speak of $L_{p}$ estimators but of $L_{p}$ aggregators.

5. We mention the work of Elkind et al. (2020) who study deliberative coalition building around candidates embedded in a metric space; indeed, the aggregation methods proposed here can produce candidates that may seem, based on the metric space, to be better for a majority of the voters, but still leave the decision to the voters whether they are indeed better or not. 
Table 1: Summary of our main results. Each block of rows corresponds to a different application, while each row corresponds to a different aggregation method. For each application we consider existence, uniqueness, majoritarity, monotonicity, and the computational complexity of each aggregation method. (Note that a "no" in the table means that the property is not guaranteed to hold for all instances; indeed, it might hold for some.)

\begin{tabular}{|c|c|c|c|c|c|c|}
\hline Setting & Aggregation & Solution & Unique & Complexity & Majoritarian & Monotonicity \\
\hline Plurality & Condorcet & Plurality & no & linear & yes & yes \\
\hline Elections & $\widetilde{L_{p}}(V)$ & Plurality & no & linear & yes & yes \\
\hline $1 \mathrm{D}$ & Condorcet & Median & for $n \in \mathbb{N}_{\text {odd }}$ & linear & yes (odd) & yes (odd) \\
\hline $\begin{array}{l}\text { Single } \\
\text { Winner }\end{array}$ & $\widetilde{L_{p}}(V)$ & $\begin{array}{c}\text { Median for } p=1 \\
\text { Average for } p=2 \\
\text { Mid-range for } p=\infty\end{array}$ & yes (for $p=1$; if convex) & $\begin{array}{l}\text { linear for } p=1,2, \infty \\
\text { efficient for } p \neq 1,2, \infty\end{array}$ & for $p=1$ (odd) & for $p=1$ (odd) \\
\hline Continuous & Condorcet & no & no & efficient & yes & yes \\
\hline Budgeting & $L_{p}(V)$ & yes & yes & $\begin{array}{c}\text { linear for } p=2 \\
\text { efficient for } p \neq 2\end{array}$ & only for $p=1$ & no \\
\hline Social & Condorcet & no & no & $?$ & yes & yes \\
\hline $\begin{array}{l}\text { Welfare } \\
\text { Functions }\end{array}$ & $\widetilde{L_{p}}(V)$ & yes & no & NP-hard & only for $p=1$ & for $p=1$ \\
\hline Committee & Condorcet & no & no & (Co)NP-hard & yes & yes \\
\hline Elections & $L_{p}(V)$ & yes & for $p=1, n \in \mathbb{N}_{\text {odd }}$ & $\begin{array}{c}\text { linear for } p=1 \\
\text { NP-hard for } p>1\end{array}$ & only for $p=1$ & only for $p=1$ \\
\hline Participatory & Condorcet & no & no & NP-hard & yes & yes \\
\hline Legislation & $L_{p}(V)$ & yes & no & NP-hard for $p=1, \infty$ & only for $p=1$ & only for $p=1$ \\
\hline
\end{tabular}

\section{Plurality Elections}

We apply our model to the standard, general model of single-winner elections (Arrow, 1951; Sen, 1986; Duncan, 1958), in which the aggregation method decides on a single winner. Specifically, we consider the categorical setting, with no structure among the alternatives. Thus, in this setting we have an underlying equidistant set of alternatives, and the task is to aggregate voter preferences to select one of these alternatives. In a sense, this is the simplest social choice setting which we present also as a warm-up.

Model Let $A$ be the underlying set of alternatives and notice that the set of possible outcomes of a single-winner election over $A$ is exactly $A$. Thus, we consider the metric space $(X, d)$ where $X=A$. Here we assume no structure or dependencies between the alternatives, hence we define $d$ to be the discrete metric, i.e., for each $x \neq y \in X, d(x, y)=1$, and $d(x, x)=0$.

For this simple scenario, Condorcet and $L_{p}$ aggregation for any $1 \leq p \neq \infty$ essentially boil down to Plurality, while $L_{\infty}(V)$ equals Plurality for unanimous elections, but otherwise selects all alternatives as co-winners.

Proposition 1. In the application to Plurality elections, Plurality is both Condorcet-consistent and minimizes $L_{p}$, for any $p \geq 1$.

Proof. For each $x \in X$, let $V(x):=\left|\left\{v_{i} \in V: v_{i}=x\right\}\right|$ denote the number of votes for $x$ among the votes $V=\left\{v_{1}, \ldots, v_{n}\right\}$. Let $z \in X$ be a Plurality winner, i.e., every $x \in X$ satisfies $V(x) \leq V(z)$. Then, for any $x \in X$, it follows that:

- First Item: $V(x \succ z)=V(x) \leq V(z)=V(z \succ x)$, 
- Second Item: $\sum_{i \in[n]} d\left(v_{i}, x\right)^{p}=n-V(x) \geq n-V(z)=\sum_{i \in[n]} d\left(v_{i}, z\right)^{p}$.

The first item above completes the proof for Condorcet aggregation, when noticing that strict equality follows if a Condorcet winner exists. The second item above completes it for $L_{p}$ aggregation for $1 \leq p \neq \infty$. For $p=\infty$, notice that $z \in L_{\infty}(V)$ for unanimous elections (in which all voters vote the same); however, as soon as there are at least two different ideal elements, we have $\max _{i}\left\{d\left(x, v_{i}\right)\right\}=1$ for every $x \in X$, hence $L_{\infty}(V)=X$, thus all alternatives tie as co-winners; in particular, the Plurality winner as well.

We note that, while $L_{\infty}(V)$ does not contain much information here, its reduced form $\widetilde{L_{\infty}}(V):=\lim _{p \rightarrow \infty} L_{p}(V)$ is restricted to the plurality winners in $X$. The following corollary is immediate.

Corollary 1. In the application to Plurality elections, both Condorcet aggregation and $L_{p}(V)$ exist for any $p \geq 1$, but are not always unique. Furthermore, they are monotonic, majoritarian, and may be computed in linear time.

Proof. Uniqueness is not guaranteed in situations where more than one alternative gets the highest number of votes. Monotonicity holds as a Plurality winner remains so whenever ballots are changed to it. Majoritarity holds as a majority of ideal elements on one alternative would make it the Plurality winner. Plurality can be computed in linear time by summing the tally of each alternative.

\section{One-Dimensional Single-Winner Elections}

Next we consider a different model of single-winner elections, corresponding to choosing a parameter in a 1-dimensional space (say, a price for a commodity or a tax rate) or political elections over a single political axis. We assume that some subset $X \subseteq \mathbb{R}$ is given as the set of alternatives. As is the case with Plurality elections (Section 4), the task here is to select a single alternative as a winner.

Model. We consider a metric space $(X, d)$, where $X \subseteq \mathbb{R}$ is a set of real numbers, and $d$ corresponds to the absolute distance; i.e., for each $x, y \in X, d(x, y)=|x-y|$ (recall that each $x \in X$ corresponds to a real number). For the ease of notation, we shall order the ideal elements in $V$ by their magnitude, i.e., $v_{1} \leq v_{2} \leq \ldots \leq v_{n}$. We begin with a simple characterization of Condorcet winners and $L_{p}$ aggregation for $p \in\{1,2, \infty\}$ in this setting.

Observation 1. For the application to 1-dimensional single-winner elections, the Condorcet aggregation and the $L_{1}$ aggregation are the median of $V .{ }^{6}$ Furthermore, if $X \subseteq \mathbb{R}$ is convex, then $L_{2}(V)=\frac{1}{n} \sum_{i} v_{i}$ (the average of $V$ ), and $L_{\infty}(V)=\frac{v_{1}+v_{n}}{2}$ (its mid-range).

Proof. For Condorcet aggregation, the result follows Black's celebrated Median Theorem (Black, 1948). The results for $L_{p}(V)$ are folklore (Pennecchi \& Callegaro, 2006).

When $X \subseteq \mathbb{R}$ is compact, the existence of $L_{p}$ aggregation for other values of $p$ follows since $\sum_{i \in[n]}\left|v_{i}-x\right|^{p}$ is continuous, and thus must obtain a minimum in $X$. Furthermore,

6. If there are two medians, then any point between them, including them, is a (weak) Condorcet winner and also minimizes $L_{1}$. 
if $X \subseteq \mathbb{R}$ is convex and $1<p \leq \infty$, this function is strictly convex and thus obtains a unique minimum. It follows that the aggregation element for convex domains may be efficiently computed via gradient descent methods. Otherwise, if $X$ is not convex, then an efficient algorithm can be realized by first computing $L_{p}^{c o n v}(V)$, the aggregated element in $\mathbb{R}$ (regardless of $X$ ). It follows that $L_{p}(V)$ is obtained in one or both elements in $X$ closest to $L_{p}^{c o n v}(V)$, i.e., either $\max \left\{x \in X: x \leq L_{p}^{c o n v}(V)\right\}$ or $\min \left\{x \in X: x \geq L_{p}^{\operatorname{conv}}(V)\right\}$. We thus conclude the following. ${ }^{7}$

Observation 2. For the application to 1-dimensional single-winner elections, Condorcet aggregation and $L_{p}$ aggregation exist wrt. any compact set of alternatives $X \subseteq \mathbb{R}$. These aggregation rules can be efficiently computed via gradient descent schemes ( $L_{1}$ aggregation and Condorcet aggregation can also be computed in linear time, as they are equivalent to the median). If $X \subseteq \mathbb{R}$ is convex, then $L_{p}(V)$ is unique for every $1<p \leq \infty$.

Remark 1. The median $L_{1}(V)$ is not necessarily unique when $|V|=n$ is even, however its reduced form $\widetilde{L_{1}}(V):=\lim _{p \rightarrow 1} L_{p}(V)$ is indeed unique on convex domains. Intuitively, we use the reduced form to arrive at rules that have less tied winners; and thus, in particular, are more resolute. Note the reduced form is not always resolute (i.e., has a unique winner), e.g., the case of $p=\infty$ presented in Section 4 , in which $\widetilde{L_{\infty}}(V) \subseteq L_{\infty}(V)$ is shown to reduce the aggregation to plurality votes but is not necessarily unique).

To get some more intuition regarding the behavior of $L_{p}$ aggregation, consider the following example.

Example 1. Consider two vote distributions on $X=[-1,1]$, namely $V, V^{\prime}$ as defined in Figure 1. For simplicity, assume $n$ is odd; the plot in Figure 1 is for $n=101$. These distributions correspond to the influence of a single voter who votes 1 wrt. consensus (in $V$ ) and symmetric polarization $\left(V^{\prime}\right)$ for the rest of the votes. The blue dashed curve corresponds to $L_{p}(V)=\frac{1}{1+n^{1 /(p-1)}}$, while the red curve corresponds to $L_{p}\left(V^{\prime}\right)=\frac{1-\left(\frac{n}{n+2}\right)^{1 /(p-1)}}{1+\left(\frac{n}{n+2}\right)^{1 /(p-1)}}$. Note that the curves intersect at $p=2$ as $L_{2}(V)=\frac{1}{n}$ for both vote distributions. Figure 1 describes the outcome of the voting rule $\mathcal{R}(V)=L_{p}(V)$ as a function of $p$.

Corollary 2. For the application to 1-dimensional single-winner elections, Condorcet aggregation and $L_{p}$ aggregation are not always unique. The reduced form $\widetilde{L_{1}}(V)$ is unique on convex domains.

Next we consider monotonicity and majoritarity.

Proposition 2. For the application to 1-dimensional single-winner elections, Condorcet aggregation and $L_{1}(V)$ are majoritarian and monotonic for odd number of voters, but not monotonic for even number of voters, while $L_{p}$ aggregation for $p>1$ is neither monotonic nor majoritarian.

Proof. Condorcet aggregation and $L_{1}(V)$ both choose the median point. Thus, majoritarity holds as having a majority of the ideal points on one element would make it be the median.

7. To avoid issues of number precision (e.g., real numbers) we assume that the input is given in a nonsuccinct way; thus, in particular, computing max can be done efficiently. 


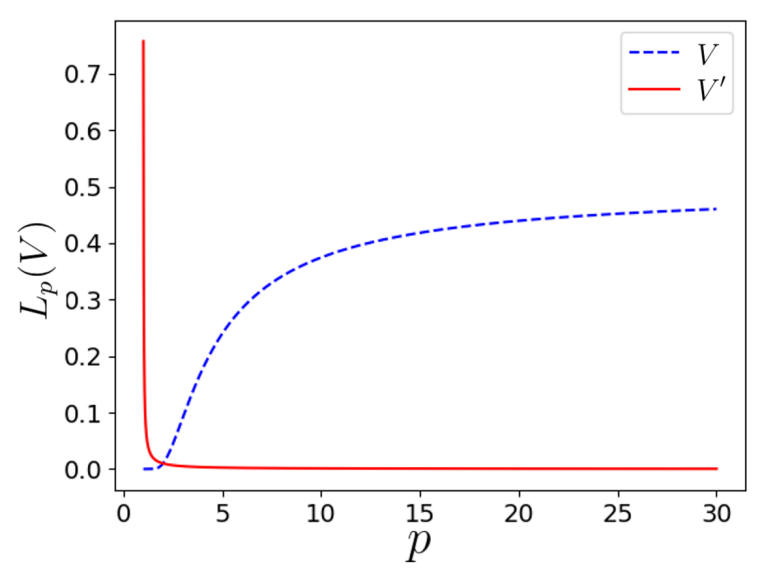

$$
\begin{gathered}
V= \begin{cases}0 & v_{1}, \ldots, v_{n-1} \\
1 & v_{n}\end{cases} \\
V^{\prime}= \begin{cases}-1 & v_{1}, \ldots, v_{(n-1) / 2} \\
1 & v_{(n-1) / 2+1}, \ldots, v_{n}\end{cases}
\end{gathered}
$$

Figure 1: The influence of outliers under $L_{p}$ aggregation as a function of $p$; see Example 1.

For odd number of voters, the median is indeed a point, thus monotonicity holds as moving elements to the median would not change the median. For even number of voters, there is originally not only a single median, thus monotonicity (as defined) is not satisfied.

For $L_{p}$ aggregation for $p>1$, the distribution $V$ in Figure 1 provides a counterexample to majoritarity, as a majority of voters choose 0 , but $L_{p}(V)>0$ for all $p>1$.

Monotonicity does not hold for similar reasons: in the same vote distribution $V$, shifting the voter $v=1$ to the aggregation point $L_{p}(V)$ results in a new vote distribution which aggregation point is strictly lower then $L_{p}(V)$ for all $p>1$.

\section{Continuous Participatory Budgeting}

Here we apply our general model to a continuous model of budgeting. Several budgeting methods exist in the literature (Faliszewski \& Talmon, 2018; Aziz et al., 2018b; Brandl, Brandt, Peters, Stricker, \& Suksompong, 2019); we also mention related work on probabilistic social choice (Brandt, 2017). Here we concentrate on a model, termed continuous participatory budgeting, in which fractional funds shall be allocated to several divisible items; this is in contrast to the indivisible model of participatory budgeting (Cabannes, 2004), in which items come with cost, and an item cannot be partially funded, see also the discussion by Aziz and Shah (2020): ${ }^{8}$ Specifically, individual financial statement items are grouped by cost centers or departments, and there is a given, fixed budget limit. In contrast to related models (Garg et al., 2017; Fain, Goel, \& Munagala, 2016), we do not assume a fixed set of alternatives provided to the agents to choose from. Rather, every agent may propose a set of alternatives, (represented, e.g., by a string) and an allocation of funds between them.

Model. We assume a fixed budget, shared by a set of agents $V$. Each agent $v \in V$ proposes a finite set of alternatives $A_{v}$ and a probability vector $v \in \mathbb{R}^{\left|A_{v}\right|}$ that corresponds to a distribution of funds among these alternatives (note that we use the term probability vector to mean a vector whose entries sum to one; in particular, we do not assume any

8. This model is sometimes also referred to as Pie Chart voting (Peters, 2019). 
probability process nor an underlying stochastic process; furthermore, the $A_{v}$ 's need not be disjoint, so several voters can support the same alternative). Let $A=\bigcup_{v} A_{v}$ denote the (finite) set of alternatives proposed by all agents. The main aim is to decide how to distribute the budget among the alternatives. Denoting $|A|=m$, the set of ideal elements consists of the $(m-1)$-dimensional simplex

$$
\Delta_{m-1}:=\left\{\left(w^{1}, \ldots, w^{m}\right) \in \mathbb{R}^{m} \mid w^{i} \geq 0 \text { and } \sum_{i} w^{i}=1\right\} .
$$

Note that the vote of each agent $v$ is naturally embedded in $\Delta_{m-1}$ via $v \mapsto\left(v^{1}, \ldots, v^{m}\right)$, $v^{i}=0$ for $i \notin A_{v}$. In order to define distances in this context, it is natural ${ }^{9}$ to consider the metric induced by the Euclidean norm

$$
d(x, y)=\|x-y\|=\left(\sum_{i}\left|x^{i}-y^{i}\right|^{2}\right)^{1 / 2}
$$

Example. Assume a joint budget is shared by the agents $V=\{1,2,3\}$. The first proposes to spend it all on beer, with $A_{1}=\{$ beer $\}$. The second proposes to purchase tea and cookies (equal funds for both), with $A_{2}=\{t e a, c o o k i e s\}$, and the third proposes to spend equal funds over $A_{3}=\{$ beer,tea,cookies $\}$. Then, $A=\{$ beer,tea,cookies $\}$ and the votes are given by $v_{1}=(1,0,0), v_{2}=\left(0, \frac{1}{2}, \frac{1}{2}\right), v_{3}=\left(\frac{1}{3}, \frac{1}{3}, \frac{1}{3}\right) \in \Delta_{2}$. As the Euclidean norm imposes a metric structure over $\Delta_{2}$, this example fits the framework introduced in 3, implying that Condorcet winners and $L_{p}$ aggregators are natural solution concepts in this setting.

Condorcet winners. The analysis of Condorcet winners in Euclidean spaces has been widely studied in the literature, and is generally referred to as a spatial model (Enelow \& Hinich, 1984). In fact, applying Plott's theorem (Plott, 1967), provides a full characterization of Condorcet winners under the metric imposed by the Euclidean norm:

Corollary 3. In this setting, Condorcet winners do not always exist, and their existence can be determined in polynomial time.

Furthermore, as follows from (Enelow \& Hinich, 1984), a Condorcet winner exists with probability that tends to zero with a large number of voters, and the Condorcet cycle usually spans over almost the entire domain.

$L_{p}$ aggregators. In contrast to Condorcet winners, since $f(x)=\sum_{i \in[n]}\left\|x-v_{i}\right\|^{p}$ is continuous over the compact domain $\Delta_{m-1}$, it follows that there always exists an element in $X=\left(\Delta_{m-1},\|\cdot\|_{p}\right)$ that minimizes $f$. Furthermore, when the ideal elements $v_{1}, \ldots, v_{n}$ are not collinear (i.e., they do not lie on the same line), it holds that $f$ is positive and strictly convex in $\Delta_{m-1}$, hence obtains a unique minimum; we refer the reader to the work of Vardi and Zhang (2000) for elaborate discussion for the case of $p=1$.

Corollary 4. $L_{p}$ aggregation always exists, and it is unique (except for the case of $p=1$ with respect to an even number of ideal elements that are collinear). In contrast, Condorcet aggregation usually does not exist and is not necessarily unique.

9. Other options exist, such as, e.g., considering metrics induced by other norms. 
Remark 2. We note that the $L_{1}$-estimator in this setting is the geometric median of $v_{1}, \ldots, v_{n}$ (sometimes called the Fermat or the Torricelli point), and the $L_{2}$-estimator is the center of mass of the given ideal elements, which corresponds to the average in every coordinate.

The situation here generalizes the situation of 1-dimensional Euclidean elections described in Section 5, as a 1-simplex is a 1-dimensional Euclidean space. From similar reasons as discussed in Section 5 (specifically, the convexity and compactness of $\Delta_{m-1}$ ), efficient computation for $L_{p}$ aggregation follows by using gradient descent.

Corollary 5. $L_{p}$ aggregation can be computed efficiently using gradient descent.

Majoritarity holds for Condorcet aggregation and for $L_{1}$ aggregation.

Proposition 3. For the application to continuous budgeting, majoritarity holds for $L_{1}$ but not for $L_{p}$ for $p>1$. No $p \geq 1$ satisfies monotonicity. Condorcet aggregation satisfies both monotonicity and majoritarity.

Proof. For $p>1$ majoritarity fails due to Proposition 2. For $p=1$, majoritarity holds as any deviation from the majority ideal element would increase the distance from the majority voters, while, following the triangle inequality, the decrease from other voters would not suffice to account for the increase. Monotonicity is not satisfied even for $p=1$ (e.g., consider a triangle).

\section{Social Welfare Functions}

We consider social welfare functions, where the input for an aggregation method is a set of $n$ linear orders and the output of an aggregation method is another linear order. This setting is the same as the classical one introduced in Arrow's impossibility theorem (Arrow, 1951), only that we are considering it in the framework of metric aggregation. A relevant metric space in this setting is the set of all permutations $X=S_{A}$ over a set of the $|A|$ underlying alternatives, equipped with the metric $d$ induced by swaps of consecutive alternatives (other options are natural for future study).

Example 2. Consider a toy example with $A=\{a, b, c\}, V=\left\{v_{1}, v_{2}, v_{3}\right\}$ with $v_{1}: a \succ b \succ c$, $v_{2}: a \succ b \succ c$, and $v_{3}: b \succ a \succ c$. It can be verified, e.g., that the $L_{\infty}$ solution here corresponds to $a \succ b \succ c$.

Observation 3. For the application to social welfare functions, $L_{p}$ aggregation for any $1 \leq p \leq \infty$ always exist but are not always unique. Condorcet aggregation does not always exist.

Proof. Being the minimum of a continuous function over a compact domain, the existence of $L_{p}$ aggregators in this setting is guaranteed for all $1 \leq p \leq \infty$. To see why $L_{p}(V)$ is not unique, consider a finite set of alternatives $A$ and $|A|$ ! voters, each of which corresponds to a different permutation over $A$. By symmetry considerations, $L_{p}(V)=S_{A}$ for every $1 \leq p \leq \infty$.

For Condorcet, consider $A=\{a, b, c\}$ with votes $v_{1}: a \succ b \succ c, v_{2}: b \succ c \succ a$, and $v_{3}: c \succ a \succ b$. Then, e.g., $a \succ b \succ c \rightarrow a \succ c \succ b \rightarrow b \succ c \succ a \rightarrow b \succ a \succ c \rightarrow c \succ a \succ b \rightarrow$ 
$c \succ b \succ a \rightarrow a \succ b \succ c$ is a cycle (that is, e.g., more voters prefer $a \succ b \succ c$ to $a \succ c \succ b$, and so on along the cycle; as the cycle spans the whole space, it implies in particular that an undominated element does not exist).

Observation 4. In the application to social welfare functions, the $L_{1}$ aggregation element is the Kemeny ranking and thus $L_{1}$ aggregation is NP-hard. ${ }^{10}$

Proof. Given $n$ ordinal ballots (i.e., $n$ linear orders) over an underlying set $A$ of alternatives, a Kemeny ranking is a linear order which minimizes the sum of distances to the $n$ linear orders, where the distance is defined via swap distance, thus equivalent to minimizing the sum of absolute distances; see, e.g., the work of Hemaspaandra et al. (2005). Kemeny is NP-hard (Bartholdi, Tovey, \& Trick, 1989); it is in fact harder (Hemaspaandra et al., 2005).

As for $L_{\infty}$, it is equivalent to the NP-hard Adjacent Element Swap Centre PerMUtATion problem (Popov, 2007, Corollary 1); thus $L_{\infty}$ is NP-hard here as well. This fact also follows from Biedl et al. (2009).

Corollary 6. For this setting, $L_{\infty}$ aggregation is NP-hard.

The following result shows that, for this setting, $L_{p}$ is NP-hard for any $p \geq 1$.

Theorem 1. For this setting, $L_{p}$ aggregation is $N P$-hard for any $1<p<\infty$.

Proof. We reduce the problem of deciding if an aggregation element has $L_{1}$ distance at most $d$ to the problem of deciding if an aggregation element has $L_{p}$ at most $d^{\prime}$, for $1<p<\infty$. The proof then follows as $L_{1}$ aggregation is NP-hard (Observation 4).

For simplicity of presentation, we describe the proof for the case of 3 voters; but the proof generalizes in a straightforward way to more voters. Let $\mathcal{A}$ be a set of alternatives, and let $V=\{a, b, c\}$ be the voters; i.e., each of $a, b$, and $c$, is a permutation over $\mathcal{A}$. Let $x^{*}$ be the $L_{1}$ aggregation element of $V$. We reduce the problem of deciding whether the $L_{1}$ distance of $x^{*}$ is below some threshold to the problem of deciding whether the optimal distance of the $L_{p}$ aggregation element of the election we describe next is below some other threshold. In the context of $L_{p}$ aggregation, given two permutations $x, y$, we write $d_{p}(x, y)=d(x, y)^{p}$ and given a set of permutations $Y, d_{p}(x, Y)=\sum_{y \in Y} d_{p}(x, y)$ (recall that the $L_{p}$ aggregation point is the permutation $x$ minimizing $\left.d_{p}(x, V)\right)$.

The reduced election has a set $\mathcal{A}_{1} \cup \mathcal{A}_{2} \cup \mathcal{A}_{3}$ of alternatives, where sets $\mathcal{A}_{i}$ are three (distinct) copies of the set $\mathcal{A}$. We write $a_{i}$ (resp. $b_{i}$ or $c_{i}$ ) for the copy of $a$ (resp. $b$ or $c$ ) over set $\mathcal{A}_{i}$, with $i \in\{1,2,3\}$, and $V_{i}=\left\{a_{i}, b_{i}, c_{i}\right\}$. The reduced instance has a set $V^{\prime}=\{A, B, C\}$ of voters, such that:

$$
\begin{aligned}
& A=a_{1} \succ b_{2} \succ c_{3} \\
& B=b_{1} \succ c_{2} \succ a_{3} \\
& C=c_{1} \succ a_{2} \succ b_{3}
\end{aligned}
$$

10. As a decision problem, we refer to the problem of deciding whether an element with at most some given $L_{1}$ distance exists. 
Recall that $x^{*}$ is the $L_{1}$ aggregation element of $V$, write $x_{i}^{*}$ for its copy over set $\mathcal{A}_{i}$. Consider the permutation

$$
X^{*}=x_{1}^{*} \succ x_{2}^{*} \succ x_{3}^{*} .
$$

Below we show that $X^{*}$ minimizes the $L_{p}$ distance, and that only strings of this form do (i.e., more generally, using any three optimal $L_{1}$ aggregation elements of $V$ instead of three copies of $x^{*}$ ). To show this, consider an arbitrary permutation $X$ (in the metric space of the reduced election).

First note that:

$$
\begin{aligned}
d_{p}\left(X^{*}, V^{\prime}\right)= & \left(d\left(x_{1}^{*}, a_{1}\right)+d\left(x_{2}^{*}, b_{2}\right)+d\left(x_{3}^{*}, c_{3}\right)\right)^{p} \\
& +\left(d\left(x_{1}^{*}, b_{1}\right)+d\left(x_{2}^{*}, c_{2}\right)+d\left(x_{3}^{*}, a_{3}\right)\right)^{p} \\
& +\left(d\left(x_{1}^{*}, c_{1}\right)+d\left(x_{2}^{*}, a_{2}\right)+d\left(x_{3}^{*}, b_{3}\right)\right)^{p} \\
= & 3 \cdot\left(d\left(x^{*}, a\right)+d\left(x^{*}, b\right)+d\left(x^{*}, c\right)\right)^{p} \\
= & 3 \cdot\left(d_{1}\left(x^{*}, V\right)\right)^{p} .
\end{aligned}
$$

Consider now any aggregation element $X$. Observe that $X$ is of the form $X=y_{1} \succ$ $y_{2} \succ y_{3}$, where $y_{i}$ is a permutation over $A$, but $y_{1}, y_{2}, y_{3}$ are not necessarily copies of each other. Otherwise, there would be a preference of the form $v \succ u$ with $u \in \mathcal{A}_{i}$ and $v \in \mathcal{A}_{j}$ with $i<j$, and swapping $u$ and $v$ would only improve the $L_{p}$ distance.

So, assume that $X$ is of the form

$$
X=y_{1} \succ y_{2} \succ y_{3} .
$$

Furthermore, we assume that $X$ does not only use $L_{1}$ aggregation elements of (copies of) $V$, i.e. $d_{1}\left(y_{1}, V_{1}\right)+d_{1}\left(y_{2}, V_{2}\right)+d_{1}\left(y_{3}, V_{3}\right)>3 \cdot d_{1}\left(x^{*}, V\right)$.

Next we compute the $d_{p}$ distance between $X$ and the electorate $V^{\prime}$, that is $d_{p}\left(X, V^{\prime}\right)$ :

$$
\begin{aligned}
d_{p}\left(X, V^{\prime}\right)= & d(X, A)^{p}+d(X, B)^{p}+d(X, C)^{p} \\
= & \left(d\left(y_{1}, a_{1}\right)+d\left(y_{2}, b_{2}\right)+d\left(y_{3}, c_{3}\right)\right)^{p} \\
& +\left(d\left(y_{1}, b_{1}\right)+d\left(y_{2}, c_{2}\right)+d\left(y_{3}, a_{3}\right)\right)^{p} \\
& +\left(d\left(y_{1}, c_{1}\right)+d\left(y_{2}, a_{2}\right)+d\left(y_{3}, b_{3}\right)\right)^{p} \\
= & u^{p}+v^{p}+w^{p},
\end{aligned}
$$

where the first equality is by definition; the second equality follows from the form of $X$; and the third is simply by defining $u, v$, and $w$ (as notation).

Now we have the following:

$$
\begin{aligned}
u+v+w= & d\left(y_{1}, a_{1}\right)+d\left(y_{2}, b_{2}\right)+d\left(y_{3}, c_{3}\right) \\
& +d\left(y_{1}, b_{1}\right)+d\left(y_{2}, c_{2}\right)+d\left(y_{3}, a_{3}\right) \\
& +d\left(y_{1}, c_{1}\right)+d\left(y_{2}, a_{2}\right)+d\left(y_{3}, b_{3}\right) \\
= & d\left(y_{1}, a_{1}\right)+d\left(y_{1}, b_{1}\right)+d\left(y_{1}, c_{1}\right) \\
& +d\left(y_{2}, a_{2}\right)+d\left(y_{2}, b_{2}\right)+d\left(y_{2}, c_{2}\right) \\
& +d\left(y_{3}, a_{3}\right)+d\left(y_{3}, b_{3}\right)+d\left(y_{3}, c_{3}\right) \\
= & d_{1}\left(y_{1}, V_{1}\right)+d_{1}\left(y_{2}, V_{2}\right)+d_{1}\left(y_{3}, V_{3}\right) \\
> & 3 \cdot L_{1}\left(x^{*}, V\right),
\end{aligned}
$$


where the first equality is by definition of $u, v$, and $w$; the second equality is by rearranging terms; the third equality is by definition of $V_{i}$ and of $d_{1}$; and the (last) inequality is from the fact that one of $y_{1}, y_{2}, y_{3}$ is not optimal.

Next we mention a folklore algebraic inequality on generalized means (Bullen, 2013), stating that for positive integers $n_{1}, \ldots, n_{k}$ and $p>1$,

$$
\frac{1}{k} \sum_{i \in[k]} n_{i} \leq\left(\frac{1}{k} \sum_{i \in[k]} n_{i}^{p}\right)^{\frac{1}{p}} .
$$

In other words, for any $m$, we have that

$$
\sum_{i \in[k]} n_{i} \geq k \cdot m \Rightarrow \sum_{i \in[k]} n_{i}^{p} \geq k \cdot m^{p} .
$$

Using this algebraic inequality for $k=3$, we have that, since $u+v+w>3 \cdot L_{1}\left(x^{*}, V\right)$, then $L_{p}\left(X, V^{\prime}\right)=u^{p}+v^{p}+w^{p}>3 \cdot L_{1}\left(x^{*}, V\right)^{p}=L_{p}\left(X^{*}, V^{\prime}\right)$.

We conclude that $X^{*}$ is an $L_{p}$ aggregation element of $V^{\prime}$, and that any other aggregation element must be a concatenation of three $L_{1}$ aggregation elements of $V$. Thus, to find the $L_{p}$ aggregation element, one cannot avoid finding the $L_{1}$ aggregation element.

We conjecture that also Condorcet aggregation is NP-hard for this setting, however proving it seems to be quite tricky.

Conjecture 1. Condorcet aggregation for this setting is NP-hard.

The next statement shows that $L_{p}$ aggregation is not monotone for $p=\infty$.

Proposition 4. $L_{\infty}$ is not monotone.

Proof. The proof follows from the one-dimensional case, in particular, from Proposition 2 as we can devise instances of social welfare functions that would correspond to one-dimensional lines.

Consider, for example, two ideal elements, $v_{1}=a \succ b \succ c \succ d \succ e$ and $v_{2}=e \succ a \succ b \succ$ $c \succ d$. Then, observe that the ranking $w=a \succ b \succ e \succ c \succ d$ satisfies $w \in L_{\infty}(V)$; however, changing $v_{2}$ to vote as $w$ would result in a new aggregation element $a \succ b \succ c \succ e \succ d$. Notice that the instance described above indeed simulates a one dimensional line (containing five points, where $v_{1}$ corresponds to the right-most point, while $v_{2}$ corresponds to the leftmost point).

Next we consider majoritarity.

Proposition 5. Condorcet aggregation and $L_{1}$ aggregation are majoritarian for the application to social welfare functions, while $L_{p}$ aggregation for $p>1$ are not.

Proof. For Condorcet aggregation, majoritarity always holds, as an ideal point with a strict voter majority would not be pairwise beaten by any other ideal point.

For $L_{1}$ aggregation, consider a linear order $x$ corresponding to the voter majority's choice and, intuitively, observe that deviating from this linear order would increase the sum 
of distances. More formally, consider some other ideal element $y \neq x$, of distance $d(x, y)$ from $x$. We first observe that if $d(x, y)=1$, then the sum of distances from all voters to $y$ is strictly no smaller than the sum of distances from all voters to $x$ :

$$
\sum_{i} d\left(v_{i}, x\right)=\sum_{v_{i} \neq x} d\left(v_{i}, x\right) \leq \sum_{v_{i} \neq x} d\left(v_{i}, y\right)+\sum_{v_{i} \neq x} 1<\sum_{v_{i} \neq x} d\left(v_{i}, y\right)+\sum_{v_{i}=x} 1=\sum_{i} d\left(v_{i}, y\right) .
$$

The first inequality follows from the triangle inequality, and the second from $x$ being the majority vote.

The above claim serves as the induction base for which we next prove the induction step, formally proving that, if it holds that for any $y$ whose distance from $x$ is at most $i$, then the sum of distances from all voters to $y$ cannot be smaller than the sum of distances from all voters to $x$ (the induction assumption), then the same holds for any $y$ whose distances from $x$ is exactly $i+1$. To prove the induction step, let $y$ be such with $d(x, y)=i+1$ and consider a series of consecutive swaps, starting from $y$, ending in $x$, and which is of smallest length (i.e., a shortest path in the swap distance graph). Consider the first swap from $y$ in this series/path, and denote the resulting point by $y^{\prime}$ (with $d\left(y, y^{\prime}\right)=1$ ). Now, the distance from $y^{\prime}$ to $x$ is exactly $i$, hence, by the induction hypothesis, it cannot be that the sum of distances from all voters to $y^{\prime}$ is smaller than the sum of distances from all voters to $x$. Finally, we show that the sum of distances from $y$ cannot be smaller than the sum of distances from $y^{\prime}$; this is so, as for all voters from the strict voter majority whose ideal element is $x$, the distance to $y^{\prime}$ is decreased by 1 wrt. their distance to $y$, while for all other voters, their distance from $y^{\prime}$ can be greater by at most 1 . Thus, $x$ would minimize $L_{1}$.

For $L_{p}$ aggregation with $p>1$, consider the set $\{a, b, c\}$ of underlying alternatives and $2 k-1$ voters; $k$ of which with the linear orders $a \succ b \succ c$, and $k-1$ with $c \succ a \succ b$. Then, we have that the sum of $L_{p}$ distances from $a \succ b \succ c$ (which is the majority choice) is $(k-1) 2^{p}$, while the sum of $L_{p}$ distances from $a \succ c \succ b$ is $\sum_{v \in V} 1^{p}=2 k-1$. It follows that for every $1<p<\infty$ there exists sufficiently large $k$ for which $a \succ c \succ b$ minimizes the sum of $L_{p}$ distances. The result for $p=\infty$ holds for every choice of $k$.

\section{Committee Elections}

Here we consider selecting a committee from a given set of candidates.

Model Let $A$ be the underlying set of alternatives and notice that the set of possible committees is $2^{A}$ (that is, the set of all possible subsets of $A$ ). Thus, we consider the metric space $(X, d)$ where $X \subseteq 2^{A}$; specifically, each $x \in X$ is a subset of $A, x \subseteq A$. As two specific cases, note that $X=2^{A}$ corresponds to the setting where the size of the committee is not given a priori but is decided by the aggregation method itself. This scenario was considered by Kilgour (2016), Duddy et al. (2016), and Faliszewski et al. (2017). However, denoting by $2_{k}^{A}$ the set of all $k$-size subsets over $A$, note that $X=2_{k}^{A}$ corresponds to the setting of $k$-committee selection, in which $k$ committee members shall be elected. This setting is studied quite extensively (Faliszewski et al., 2017b). As we assume no structure or dependencies between the alternatives, we do not impose any particular structure on $X$ and define $d$ to be the symmetric distance: For each $x, y \in X, d(x, y)=|x \triangle y|$ (indeed, $d(x, y)$ is the Hamming distance between the corresponding binary vectors of $x$ and $y$ ). 
First, recall the definition of the median element (Meir, 2018): Consider an election $E$ based on $n$ ideal elements $V=\left\{v_{1}, \ldots, v_{n}\right\}, v_{i} \in X$. Then, the median element is the element $x \in X$ which contains exactly those alternatives $a \in A$ for which $\mid\{v \in V: a \in$ $v\} \mid \geq n / 2$. That is, the median element is the unique element containing exactly those alternatives with weak voter majority support.

Proposition 6. For the application to committee elections, if $X=2^{A}$ then $L_{1}(V)$ chooses the median element, while if $X=2_{k}^{A}$, then $L_{1}$ aggregation is approval voting.

Proof. For $X=2^{A}$, for a set of voters $V$, the aggregated element $x=L_{1}(V)$ minimizes the expression $\sum_{v_{i} \in V}\left|x \triangle v_{i}\right|$, which equals to $\sum_{v_{i} \in V} \sum_{a \in A} \mathbb{1}_{a \in x} \triangle v_{i}$, which equals to $\sum_{a \in A} \sum_{v_{i} \in V} \mathbb{1}_{a \in x \triangle v_{i}}$. Thus, any element $y$ different from the median element $x$ would give a higher sum, as for each $a \in x \triangle y, \sum_{v_{i} \in V} \mathbb{1}_{a \in y \triangle v_{i}} \geq \sum_{v_{i} \in V} \mathbb{1}_{a \in x \triangle v_{i}}$ holds. The case where $X=2_{k}^{A}$ follows similarly.

Remark 3. The MV rule, described recently by Faliszewski et al. (2017), which elects the unique committee containing all candidates with weak majority approval, is minimizing $L_{1}$ for the setting where $X=2^{A}$. Notice that, while $M V$ is resolute, $L_{1}(V)$ is not unique for even number of voters (e.g., consider $V=\left\{v_{1}, v_{2}\right\}$ with $v_{1}:\{a\}$ and $v_{2}=\{b\}$; then, each of $\emptyset,\{a\},\{b\}$, and $\{a, b\}$ are winners $)$. For odd number of voters, though, $L_{1}(V)$ is unique.

For $X=2^{A}$, finding $L_{\infty}(V)$ is equivalent to the NP-hard Closest String problem (Frances $\&$ Litman, 1997). We also mention that it is equivalent to the variable number of winners counterpart of minimax approval voting (Brams \& Fishburn, 2007).

Corollary 7. For the application to committee elections with variable number of winners, the decision version of $L_{\infty}(V)$ (i.e., deciding whether an element with at most some given $L_{\infty}(V)$ value exists) is NP-hard.

Chen et al. (2018), using a slightly different jargon, prove that $L_{p}$ aggregation is NP-hard for all values of $p>1$.

Corollary 8. For the application to committee elections with variable number of winners, $L_{p}$ aggregation is NP-hard for any $p>1$.

While $L_{p}$ aggregation always exist for this setting, the next example demonstrates that Condorcet winners do not always exist for this application.

Example 3. Consider alternatives $A=\{a, b, c\}$ and voters $V=\left\{v_{1}, v_{2}, v_{3}, v_{4}, v_{5}\right\}$ where $v_{1}=v_{2}=\emptyset, v_{3}=\{a, b\}, v_{4}=\{a, c\}$, and $v_{5}=\{b, c\}$. Then, $\emptyset$ beats all other elements in the metric space, except $\{a, b, c\}$, but $\{a, b, c\}$ is beaten by any of $\{a\},\{b\}$, and $\{c\}$; thus, no Condorcet winner exists here.

Condorcet aggregation for this application can be phrased also as a Condorcet-consistent aggregation method for a binary combinatorial domain with top-based input, where the completion principle is based on the Hamming distance (Chevaleyre et al., 2008). Condorcet aggregation was studied by Darmann (2013) who demonstrated the computational intractability of a related problem (2013, Theorem 3.7). 
We note that for committee elections, only one committee may be a Condorcet winner: namely the one obtained by the majority voting rule, i.e. taking each alternative selected in a majority of preferences. Indeed, for any other committee, there is no strict majority against adding or removing an "unpopular" alternative. However, this is a necessary condition only, and it remains to decide if such a committee actually is a Condorcet winner.

Theorem 2. For the application to committee elections with variable number of winners, Condorcet aggregation ${ }^{11}$ is coNP-hard.

Proof. We provide a reduction from the NP-hard VerTex Cover problem. Consider a graph $G$ with vertex set denoted $A$ (with $n:=|A|$ ) and edge set $E$ (with $|E|$ ); and an even integer $k$. We first duplicate every edge in $E$ ( $E$ becomes a multi-set), and write $m$ for the (new) size of $E$. Note that this operation is safe, in the sense that a subset of vertices is a vertex cover in the original graph if, and only if, it is a vertex cover in the new one. We further assume that $G$ has no isolated vertices, is not a star, and that $n \geq k+4$.

Let the set of alternatives be $\mathcal{A}:=A \cup B$, where $B$ is a set of $k-1$ dummy alternatives. Build a set of $2 m+1$ voters $\mathcal{V}:=E \cup F \cup\{g\}$ as follows:

- $E$ is the set of edges, i.e. it is a set of $m$ voters with preference $\{u, v\} \subset A$

- $F$ is a set of $m$ voters, all approving $B$

- $g$ is a voter with preference $\mathcal{A}=A \cup B$

The majority voting winner for this election (denoted $w$ ) is the set $B$ of alternatives. Indeed, each element of $B$ appears in the preference set of $m+1$ voters, and no element in $A$ appears in $m+1$ preference sets (otherwise such an element would be a vertex incident to all edges, i.e., $G$ would be a star). We prove the following equivalence:

$w$ is not a Condorcet winner $\Leftrightarrow G$ has a vertex cover of size $k$.

We first compute the distances between $w$ and the different voters. For $e \in E, d(w, e)=$ $|B|+|e|=k+1$ (since $e$ and $B$ are disjoint). For $f \in F, d(w, f)=0$. Finally, for voter $g$, $d(w, g)=|A|=n$. Now for any other committee $h \in 2^{\mathcal{A}}, h \neq w$, we have $d(h, e)=|h|-2$ if $h$ contains $e, d(h, e)=|h|$ if $h$ contains one endpoint of $e$, and $d(h, e)=|h|+2$ otherwise. Also, $d(h, f)>d(w, f)$ for all $f \in F$, and $d(h, g)<d(w, g) \Leftrightarrow|h| \geq k$.

$\Leftarrow$. Let $h \subset A$ be a vertex cover of $G$ of size $k$ (then $h \subset \mathcal{A}$ is also a candidate committee). For any voter $e \in E$, since $h \cap e \neq \emptyset$, we have $d(h, e) \leq|h|=k<d(w, e)$; and for voter $g, d(h, g)<d(w, g)$. So all $m+1$ voters in $E \cap\{g\}$ strictly prefer $h$ over $w: w$ is not a Condorcet winner.

$\Rightarrow$. Assume that $w$ is not a Condorcet winner. Let $h \subseteq A \cup B, h \neq w$ be a committee beating $w$, i.e., a (not necessarily strict) majority of voters prefer $h$ over $w$. Since voters in $F$ strictly prefer $w$ over $h$, then all voters in $E \cup\{g\}$ must strictly prefer $h$ over $w$ (except at most one voter, in case of a tie, which is at the same distance from both). Thus, $|h|>k-1$, and $d(h, e)<k+1$ for all $e \in E$ (except for at most one $e \in E$ we may have $d(h, E)=k+1$, in case of a tie). In particular, using $d(h, e) \geq|h|-2$ for all $e$, the latter implies $|h|<k+3$.

11. in the following decision version: given a set of alternatives and voters, is there a Condorcet winner? 
First if $|h|=k-1$ (the unique equality is for voter $g$ ), then $d(h, e)<k+1$ implies that $h$ intersects every edge $e: h$ is a size- $(k-1)$ vertex cover.

Now if $|h|=k$, and $e \cap h \neq \emptyset$ for all (or all but one) edge $e$. Then $h$ covers at least $m-1$ edges, which is sufficient to be a vertex cover (since every edge has been duplicated).

Finally if $|h| \geq k+1$, then $d(h, e)=|h|-2$ for all but one edge, i.e. $e \subset h$. Thus, $h$ contains all vertices (except at most one single pending vertex) and $|h| \geq n-1$. Using $|h|<k+3$, we get $n<k+4$, a contradiction.

Next we consider majoritarity.

Observation 5. For the application to committee elections with variable number of winners, Condorcet and $L_{1}$ aggregation are both majoritarian, while $L_{p}$ aggregation for $p>1$ are not majoritarian.

Proof. For Condorcet aggregation majoritarity holds for any metric space, thus, in particular, also here. For $L_{1}$ aggregation, the claim follows as the median element would be the choice of the voter majority, and, in case of voter majority on one ideal element, it would be the median element.

For $L_{p}$ aggregation with $p>1$, choose sufficiently large odd $n$ such that $\frac{n}{n-1}<2^{p-1}$. Now, consider the set $A=\{a, b\}$ of underlying alternatives, and a set of votes given by $V=\left\{\{a\}^{(n+1) / 2},\{b\}^{(n-1) / 2}\right\}$, where the exponent of each element corresponds to the number of votes for this element.

For this instance, we have

$$
\min _{x \in X} \sum_{i \in[n]} d\left(v_{i}, x\right)^{p} \leq \sum_{i \in[n]} d\left(v_{i},\{a, b\}\right)^{p}=n
$$

In contrast,

$$
\sum_{i \in[n]} d\left(v_{i},\{a\}\right)^{p}=\frac{n-1}{2} \cdot 2^{p}=(n-1) 2^{p-1}>n .
$$

It follows that, even though the majority choice is $\{a\}$, the $L_{p}$ aggregation of this instance must be some $x \neq\{a\}$ in $X$.

The following example shows that monotonicity is not satisfied for $p>1$.

Example 4. Consider an underlying set $A=\{a, b, c, d\}$ of alternatives and ideal elements $V=\left\{v_{1}, v_{2}\right\}$ with $v_{1}=\emptyset$ and $v_{2}=A=\{a, b, c, d\}$. Then, the element $\{a, b\}$ is an aggregation point for $L_{p}$ aggregation, for $p>1$, however if $v_{2}$ moves to $\{a, b\}$ then the new aggregation element would be $\{a\}$.

Condorcet aggregation does satisfy monotonicity, as it does for all applications we consider in this paper. $L_{1}$ aggregation is monotone here as the median element would stay when moving a voter ideal element to it. 


\section{Participatory Legislation}

We consider participatory legislation (Alsina \& Martí, 2018). Clearly, plurality elections can be applied to approve a proposed legislation, or to choose among a given set of proposed legislation. Even if such a proposal is made by an elected legislation committee, such a process falls short of the egalitarian vision of the 1789 Declaration of the Rights of Man and Citizen (National Constituent Assembly, 1789, Article VI), which states that all citizens have the right of contributing to the formation of the law personally, not only via their representatives. Hence, we focus here on aggregating text drafts, e.g., to jointly write a constitution. In fact, our main motivation for this work comes from the apparent lack of literature on a participatory approach to this key social choice setting in the computational social choice community.

Model Here we have a certain alphabet $\Sigma$. The possible outcomes, as well as the possible votes, are all strings over $\Sigma$; thus, we consider a metric space $(X, d)$ where $X=\Sigma^{*}$, such that each $x \in X$ is a string over the alphabet $\Sigma$.

Various possibilities exist for defining the metric $d$. The first thing that comes to mind is to consider the Levenshtein edit distance (Levenshtein, 1966): For two ideal elements $x, y \in X$, the Levenshtein distance $d(x, y)$ is the minimum number of edit operations of the form insert (which adds a character at a certain position), delete (which deletes a character at a certain position), and swap (which swaps two consecutive characters) that shall be performed to convert $x$ into $y$. Assume that strings are documents consisting of a sequences of sentences, namely that characters are sentences. We feel that in this setting, this metric is not appropriate as it gives the same importance to the identity of the sentences as to their order. However, when writing, e.g., a constitution, the contents of the sentences in it are more important than their order. For this reason, we consider a different distance metric, namely a weighted-Levenshtein distance $d(x, y)$, which equals the minimum weight of operations that transform $x$ to $y$, with the following weighted operations: an insert operation costs 1 , a delete operation costs 1 , and a swap operation costs $1 / \ell^{2}$, where $\ell$ is the length of the longest ideal element proposed by any voter. The situation now boils down to a two-phase election: First, one shall find the set of sentences to be included in the constitution; second, one shall find the best ordering of these sentences. We consider these two phases in turn.

Phase 1: Selecting the set of sentences Here, only considering the set of sentences in each ideal element, the task is to aggregate those $n$ sets of sentences into one set of sentences. We assume that the resulting aggregated set has at most one copy of each sentence. Thus, this setting is formally equivalent to multiwinner elections with a variable number of winners, studied in Section 8.

Phase 2: Selecting the order of the selected sentences Following Phase 1, we shall order the elected sentences. This setting is formally equivalent to social welfare functions, studied in Section 7.

Combining the two phases Naturally, we get the combined hardness of the two phases: Existence is guaranteed only for $L_{p}$ aggregation; uniqueness is not guaranteed for any aggregation method; Condorcet and $L_{1}$ aggregation are majoritarian, while $L_{p}$ aggregation for $p>1$ are not majoritarian; and computational hardness follows. 
Remark 4. For $L_{1}$ aggregation, one can use the polynomial time algorithm for $L_{1}$ aggregation for multiwinner elections with variable number of winners discussed in Section 8, followed by solving Phase 2 heuristically, e.g., using one of the methods for Kemeny ranking studied by Ali and Meilă (2012).

\section{Discussion}

We proposed a general metric-based model and applied it to a broad range of social choice settings, demonstrating its applicability as providing a unifying elicitation and aggregation framework for many social choice situations. Besides the technical issues highlighted by our model, such as finding efficient aggregation algorithms for different settings and aggregation goals, we view our model and results as the theoretical foundation to be used for a providing a unified method and system for an online community to govern itself, making it possible to treat many different types of decisions to be made by community members in a similar way. In addition, the model developed in this paper has been the theoretical foundation for a study that involves deliberative decision making (Elkind et al., 2020) and paves the road for unified extensions regarding Sybil-resilient social choice (Shahaf, Shapiro, \& Talmon, 2018). More generally, the unified model presented here can serve as the basis on top of which orthogonal issues of decision making, such as Sybil-resilience, partial participation, and deliberation, can be approached in a unified way, thus making them applicable to many social choice settings at once.

Below we discuss some avenues for future research.

\subsection{Coping with NP-Hardness}

Throughout, we studied the worst-case complexity of our aggregation methods for several applications with their corresponding metric spaces, and, indeed, showed that for some applications, the combinatorial problem corresponding to identifying the winners is computationally intractable in the sense that it is an NP-hard problem.

A natural future work would be to explore the possibility of computing such winners in practice, through the use of efficient heuristics and metaheuristics, as well as employing general purpose solvers (such as SAT solvers and ILP solvers), and to experimentally evaluate their performance via computer-based simulations on real-world data and on synthetic data. Indeed, it might be still the case that, some of the cases for which we derive NP-hardness results can still be solved quite well in practice, enabling their use in practice.

\subsection{Further Settings}

Further social choice settings shall be realized in our general model. In particular, further distance measures, such as, e.g., those described by Schiavinotoo and Stützle (2007) can be applied. Related, our aggregation methods for participatory legislation only take into account syntactic distance, but not semantic distance. An intriguing avenue for future research would be to apply NLP methods which take into account the semantics of the aggregated text drafts.

Furthermore, and also related to the fact that in our model voters provide ideal points from which their preferences is induced, an important direction for future work is to some- 
how lift this restriction, perhaps by allowing for different, possibly iterative, elicitation method that would allow voters to specify more involved preferences (for example, preferences regarding project interactions in participatory budgeting (Jain, Sornat, \& Talmon, 2020), succinctly).

\subsection{Extensions}

A major advantage of utilizing a unified framework, capturing many social choice settings at once, is that extensions and generalizations can be designed in a unified way, making them applicable to all such settings at once. One natural extension would be to incorporate proxy voting (Miller, 1969) and liquid democracy (Behrens, Kistner, Nitsche, \& Swierczek, 2014; Brill \& Talmon, 2018) to the model. Another natural extension would be to incorporate sybil resilience (Shahaf et al., 2018) to the model, making it applicable in situations in which not all agents participating in the process can be assumed to be genuine.

\subsection{Voting and Deliberation}

A major caveat of our model is that we infer voter preferences over the metric elements based only on their ideal elements, while it might be the case that, e.g., voters with identical ideal elements have different preferences over other elements of the metric space. This is especially problematic, e.g., when dependencies exist between committee members in multiwinner elections; where interdependent items are to be funded in participatory budgeting; or where different sentence orderings alter the meaning of the resulting text.

One remedy for this might be to incorporate Reality (the status quo) in a process that interleaves voting with deliberation; for instance, similarly in spirit to Reality-aware action plans (Shapiro \& Talmon, 2018) and to other works (Slavkovik \& Jamroga, 2016). An iterative process where the deliberation phase of each iteration can employ the aggregated element identified based on the voting phase of the last iteration, following which partici-

pants may change their votes, was presented recently (Elkind et al., 2020). The result of such an iterative process could be an aggregated element that has majority support over the status quo, or else a recognition that the status quo is presently preferred over any aggregated alternative. Deeper integration of the current work and the deliberative framework proposed by Elkind et al. (2020) may result in further progress.

\section{Acknowledgements}

A preliminary, short version of this work exists (Shahaf, Shapiro, \& Talmon, 2019). This work includes more results and further discussions.

Ehud Shapiro is the Incumbent of The Harry Weinrebe Professorial Chair of Computer Science and Biology. We thank the generous support of the Braginsky Center for the Interface between Science and the Humanities. Nimrod Talmon was supported by the Israel Science Foundation (ISF; Grant No. 630/19). 


\section{References}

Ali, A., \& Meilă, M. (2012). Experiments with Kemeny ranking: what works when?. Mathematical Social Sciences, 64(1), 28-40.

Alsina, V., \& Martí, J. L. (2018). The birth of the CrowdLaw movement: tech-based citizen participation, legitimacy and the quality of lawmaking. Analyse $\&$ Kritik, $40(2)$, $337-358$.

Anshelevich, E., Bhardwaj, O., Elkind, E., Postl, J., \& Skowron, P. (2018). Approximating optimal social choice under metric preferences. Artificial Intelligence, 264, 27-51.

Anshelevich, E., \& Postl, J. (2017). Randomized social choice functions under metric preferences. Journal of Artificial Intelligence Research, 58, 797-827.

Arrow, K. J. (1951). Social choice and individual values. Yale university press.

Aziz, H., Faliszewski, P., Grofman, B., Slinko, A., \& Talmon, N. (2018a). Egalitarian committee scoring rules. In Proceedings of IJCAI'18, pp. 56-62.

Aziz, H., Lee, B., \& Talmon, N. (2018b). Proportionally representative participatory budgeting: Axioms and algorithms. In Proceedings of AAMAS '18, pp. 23-31.

Aziz, H., \& Lee, B. E. (2019). Proportionally representative participatory budgeting with ordinal preferences. arXiv preprint arXiv:1911.00864.

Aziz, H., \& Shah, N. (2020). Participatory budgeting: Models and approaches. arXiv preprint arXiv:2003.00606.

Barbera, S., Bossert, W., \& Pattanaik, P. K. (2004). Ranking sets of objects. In Handbook of Utility Theory, pp. 893-977.

Bartholdi, J., Tovey, C. A., \& Trick, M. A. (1989). Voting schemes for which it can be difficult to tell who won the election. Social Choice and Welfare, 6(2), 157-165.

Behrens, J., Kistner, A., Nitsche, A., \& Swierczek, B. (2014). The Principles of LiquidFeedback. Interaktive Demokratie e.V.

Biedl, T., Brandenburg, F. J., \& Deng, X. (2009). On the complexity of crossings in permutations. Discrete Mathematics, 309(7), 1813-1823.

Black, D. (1948). On the rationale of group decision-making. Journal of Political Economy, $56(1), 23-34$.

Brams, S., \& Fishburn, P. C. (2007). Approval voting. Springer.

Brandl, F., Brandt, F., Peters, D., Stricker, C., \& Suksompong, W. (2019). Donor coordination: Collective distribution of individual contributions. Unpublished manuscript.

Brandt, F. (2017). Rolling the dice: recent results in probabilistic social choice. In Endriss, U. (Ed.), Trends in Computational Social Choice. AI Access.

Brandt, F., Conitzer, V., Endriss, U., Procaccia, A. D., \& Lang, J. (2016). Handbook of Computational Social Choice. Cambridge University Press.

Brill, M., \& Talmon, N. (2018). Pairwise liquid democracy. In Proceedings of IJCAI '18, pp. $137-143$.

Bullen, P. S. (2013). Handbook of Means and Their Inequalities. Springer. 
Cabannes, Y. (2004). Participatory budgeting: a significant contribution to participatory democracy. Environment and Urbanization, 16(1), 27-46.

Caragiannis, I., Nath, S., Procaccia, A. D., \& Shah, N. (2017). Subset selection via implicit utilitarian voting. Journal of Artificial Intelligence Research, 58, 123-152.

Chen, J., Hermelin, D., \& Sorge, M. (2018). On computing centroids according to the p-norms of Hamming distance vectors. arXiv preprint arXiv:1807.06469.

Chevaleyre, Y., Endriss, U., Lang, J., \& Maudet, N. (2008). Preference handling in combinatorial domains: From AI to social choice. AI magazine, 29(4), 37-46.

Darmann, A. (2013). How hard is it to tell which is a Condorcet committee?. Mathematical Social Sciences, 66 (3), 282-292.

Duddy, C., Piggins, A., \& Zwicker, W. (2016). Aggregation of binary evaluations: a Bordalike approach. Social Choice and Welfare, 46(2), 301-333.

Duncan, B. (1958). The theory of committees and elections. Cambridge University Press.

Elkind, E., Faliszewski, P., \& Slinko, A. (2015). Distance rationalization of voting rules. Social Choice and Welfare, 45(2), 345-377.

Elkind, E., Grossi, D., Shapiro, E., \& Talmon, N. (2020). Egalitarian deliberative decision making. arXiv preprint arXiv:2001.08031.

Endriss, U., \& Grandi, U. (2017). Graph aggregation. Artificial Intelligence, 245, 86-114.

Enelow, J. M., \& Hinich, M. J. (1984). The spatial theory of voting: An introduction. Cambridge University Press.

Fain, B., Goel, A., \& Munagala, K. (2016). The core of the participatory budgeting problem. In Proceedings of WINE '16, pp. 384-399.

Fain, B., Goel, A., Munagala, K., \& Sakshuwong, S. (2017). Sequential deliberation for social choice. In Proceedings of WINE '17, pp. 177-190.

Faliszewski, P., Skowron, P., Slinko, A., \& Talmon, N. (2017a). Multiwinner rules on paths from k-Borda to Chamberlin-Courant. In Proceedings of IJCAI '17, pp. 192-198.

Faliszewski, P., Skowron, P., Slinko, A., \& Talmon, N. (2017b). Multiwinner voting: A new challenge for social choice theory. In Endriss, U. (Ed.), Trends in Computational Social Choice. AI Access Foundation.

Faliszewski, P., \& Talmon, N. (2018). A framework for approval-based budgeting methods. arXiv preprint arXiv:1809.04382.

Faliszewski, P., Slinko, A., \& Talmon, N. (2017). The complexity of multiwinner voting rules with variable number of winners. arXiv preprint arXiv:1711.06641.

Feldman, M., Fiat, A., \& Golomb, I. (2016). On voting and facility location. In Proceedings of EC'16, pp. 269-286.

Filtser, A., \& Filtser, O. (2020). Plurality in spatial voting games with constant beta. arXiv preprint arXiv:2005.04799.

Frances, M., \& Litman, A. (1997). On covering problems of codes. Theory of Computing Systems, 30(2), 113-119. 
Garg, N., Kamble, V., Goel, A., Marn, D., \& Munagala, K. (2017). Collaborative optimization for collective decision-making in continuous spaces. In Proceedings of $W W W{ }^{\prime} 17$, pp. 617-626.

Gershkov, A., Moldovanu, B., \& Shi, X. (2019). Voting on multiple issues: what to put on the ballot?. Theoretical Economics, 14(2), 555-596.

Gershkov, A., Moldovanu, B., \& Shi, X. (2020). Monotonic norms and orthogonal issues in multidimensional voting. Journal of Economic Theory.

Goel, A., Krishnaswamy, A. K., Sakshuwong, S., \& Aitamurto, T. (2015). Knapsack voting. Collective Intelligence.

Goel, A., Krishnaswamy, A. K., \& Munagala, K. (2017). Metric distortion of social choice rules: Lower bounds and fairness properties. In EC'17, pp. 287-304.

Hemaspaandra, E., Spakowski, H., \& Vogel, J. (2005). The complexity of Kemeny elections. Theoretical Computer Science, 349(3), 382-391.

Jain, P., Sornat, K., \& Talmon, N. (2020). Participatory budgeting with project interactions. In Proceedings of IJCAI' '20, pp. 386-392.

Kilgour, M. (2016). Approval elections with a variable number of winners. Theory and Decision, 81(2), 1-13.

Lang, J., \& Xia, L. (2016). Voting in combinatorial domains. In Brandt, F., Conitzer, V., Endriss, U., Lang, J., \& Procaccia, A. D. (Eds.), Handbook of Computational Social Choice, chap. 9. Cambridge University Press.

Levenshtein, V. I. (1966). Binary codes capable of correcting deletions, insertions, and reversals. Soviet physics doklady.

Meir, R. (2018). Strategic Voting. M\&C.

Meir, R., Procaccia, A. D., \& Rosenschein, J. S. (2012). Algorithms for strategyproof classification. Artificial Intelligence, 186, 123-156.

Miller, J. C. (1969). A program for direct and proxy voting in the legislative process. Public Choice, 7(1), 107-113.

National Constituent Assembly (1789). Declaration of the rights of man and of the citizen. 1789.

Osborne, M. J., \& Slivinski, A. (1996). A model of political competition with citizencandidates. The Quarterly Journal of Economics, 111(1), 65-96.

Pennecchi, F., \& Callegaro, L. (2006). Between the mean and the median: the Lp estimator. Metrologia, 43(3), 213.

Peters, D. (2019). Fair Division of the Commons. Ph.D. thesis, University of Oxford.

Peters, H., van der Stel, H., \& Storcken, T. (1993). Generalized median solutions, strategyproofness and strictly convex norms. Zeitschrift für Operations Research, 38(1), 19-53.

Plott, C. R. (1967). A notion of equilibrium and its possibility under majority rule. The American Economic Review, 57(4), 787-806. 
Popov, V. Y. (2007). Multiple genome rearrangement by swaps and by element duplications. Theoretical Computer Science, 385(1-3), 115-126.

Procaccia, A. D., \& Rosenschein, J. S. (2006). The distortion of cardinal preferences in voting. In Proceedings of CIA '06, pp. 317-331.

Procaccia, A. D., \& Tennenholtz, M. (2009). Approximate mechanism design without money. In Proceedings of EC' '09, pp. 177-186.

Schiavinotto, T., \& Stützle, T. (2007). A review of metrics on permutations for search landscape analysis. Computers \& Operations Research, 34(10), 3143-3153.

Sen, A. (1986). Social choice theory. Handbook of Mathematical Economics, 3, 1073-1181.

Shahaf, G., Shapiro, E., \& Talmon, N. (2018). Sybil-resilient reality-aware voting. arXiv preprint arXiv:1807.11105.

Shahaf, G., Shapiro, E., \& Talmon, N. (2019). Aggregation over metric spaces: Proposing and voting in elections, budgeting, and legislation. In Proceedings of ADT'19.

Shapiro, E., \& Talmon, N. (2018). Incorporating reality into social choice. In Proceedings of AAMAS '18, pp. 1188-1192.

Shapiro, E. (2018). Point: foundations of e-democracy. Communications of the ACM, 61(8), $31-34$.

Skowron, P., \& Elkind, E. (2017). Social choice under metric preferences: Scoring rules and STV. In Proceedings of AAAI'17, pp. 706-712.

Slavkovik, M., \& Jamroga, W. (2016). Iterative judgment aggregation. In Proceedings of ECAI' '16, pp. 1528-1536.

Sposito, V. (1990). Some properties of Lp-estimation. Robust Regression: Analysis and Applications.

Vardi, Y., \& Zhang, C.-H. (2000). The multivariate L1-median and associated data depth. Proceedings of the National Academy of Sciences, 97(4), 1423-1426. 